Sādhanā Vol. 38, Part 1, February 2013, pp. 123-131. (C) Indian Academy of Sciences

\title{
ROI-based DICOM image compression for telemedicine
}

\author{
VINAYAK K BAIRAGI ${ }^{1, *}$ and ASHOK M SAPKAL ${ }^{2}$ \\ ${ }^{1}$ Department of Electronics Engineering, Sinhgad Academy of Engineering, \\ Pune 411048, India \\ ${ }^{2}$ Department of Electronics Engineering, College of Engineering, Pune 411005, India \\ e-mail: vbairagi@yahoo.co.in
}

MS received 12 January 2011; revised 1 November 2012; accepted 4 November 2012

\begin{abstract}
Many classes of images contain spatial regions which are more important than other regions. Compression methods capable of delivering higher reconstruction quality for important parts are attractive in this situation. For medical images, only a small portion of the image might be diagnostically useful, but the cost of a wrong interpretation is high. Hence, Region Based Coding (RBC) technique is significant for medical image compression and transmission. Lossless compression schemes with secure transmission play a key role in telemedicine applications that help in accurate diagnosis and research. In this paper, we propose lossless scalable RBC for Digital Imaging and Communications in Medicine (DICOM) images based on Integer Wavelet Transform (IWT) and with distortion limiting compression technique for other regions in image. The main objective of this work is to reject the noisy background and reconstruct the image portions losslessly. The compressed image can be accessed and sent over telemedicine network using personal digital assistance (PDA) like mobile.
\end{abstract}

Keywords. DICOM image; integer wavelet transform; lossless compression; medical image compression; region-based coding; telemedicine.

\section{Introduction}

Large amount of image data is produced in the field of medical imaging in the form of Computed Tomography (CT), Magnetic Resonance Imaging (MRI), and Ultrasound Images, which can be stored in picture archiving and communication system (PACS) or hospital information system (Miaou et al 2009). A medium scale hospital with above facilities produces on an average 5 GB to 15 GB of data (Placidi 2009); (Baeza \& Verdoy 2009). So, it is really difficult for hospitals to manage the storing facilities for the same. Moreover, such high data demand for high end network especially for transmitting the images over the network such as in telemedicine. This is significant for telemedicine scenario due to limitations of transmission medium in Information and Communication Technology (ICT) especially for rural area. Image compression is useful in, reducing the storage and transmission bandwidth requirements of medical images 
(Baeza \& Verdoy 2009). For e.g., an 8-bit grey scale image with $512 \times 512$ pixels requires more than $0.2 \mathrm{MB}$ of storage. If the image is compressed by $8: 1$ compression without any perceptual distortion, the capacity of storage increases 8 times (Ali \& Akhtar 2008). Compression methods are classified into lossless and lossy methods. In the medical imaging scenario, lossy compression schemes are not generally used. This is due to possible loss of useful clinical information which may influence diagnosis. In addition to these reasons, there can be legal issues. Storage of medical images is generally problematic because of the requirement to preserve the best possible image quality which is usually interpreted as a need for lossless compression (Sanchez et al 2009). 3D MRI contains multiple slices representing all information required about a body part.

Some of the most desirable properties of any compression method for 3D medical images include: (i) high lossless compression ratios, (ii) resolution scalability, which refers to the ability to decode the compressed image data at various resolutions, and (iii) quality scalability, which refers to the ability to decode the compressed image at various qualities or signal-to-noise ratios (SNR) up to lossless reconstruction (Sanchez et al 2009). DICOM is the most comprehensive and accepted version of an imaging communications standard. DICOM format has a header which contains information about the image, imaging modality and information about the patient (Maglogiannis \& Kormentzas 2009). The header also contains information about the type of media (CT, MRI, audio recording, etc.) and the image dimensions. Body of DICOM standard contains information objects such as medical reports, audio recordings and images. The codingdecoding algorithm must take care of other information in the DICOM file. Also, the algorithms should accept the input image in DICOM format at encoder end and produce DICOM file at decoder end.

\subsection{Region of interest}

Basic concept of Region of Interest (ROI) is introduced due to limitations of lossy and lossless compression techniques. For well-known lossless compression technique the compression ratio is approximately $25 \%$ of original size, where as for lossy encoders the compression ratio is much higher (up to 1\% also) (Baeza \& Verdoy 2009), but there is loss in the data. Now this loss may hamper some diagnostically important part of the image. Hence, there is a need of some hybrid technique which will take care of diagnostically important part (ROI) as well as will provide high compression ratio (Miaou et al 2009); (Maglogiannis \& Kormentzas 2009). The functionality of ROI is important in medical applications where certain parts of the image are of higher diagnostic importance than others. For most medical images, the diagnostically significant information is localized over relatively small regions, about 5 to $10 \%$ of total area. In such cases, these regions need to be encoded at higher quality than the background. During image transmission for telemedicine purposes, these regions are required to be transmitted first or at a higher priority (Maglogiannis \& Kormentzas 2009).

\section{Proposed method}

A CT or MRI image contains three parts, ROI (the diagnostically important part), Non-ROI image part, and the background (part other than image contents) as shown in figure 1 and its cross section in figure 2. The ROI is selected by expert radiologists. Depending on the selected part ROI-mask is generated in such a way that the foreground is totally included and the pixel values in the background are made zero. The background regions though they appear to be black in colour, they do not have zero grey level values. 


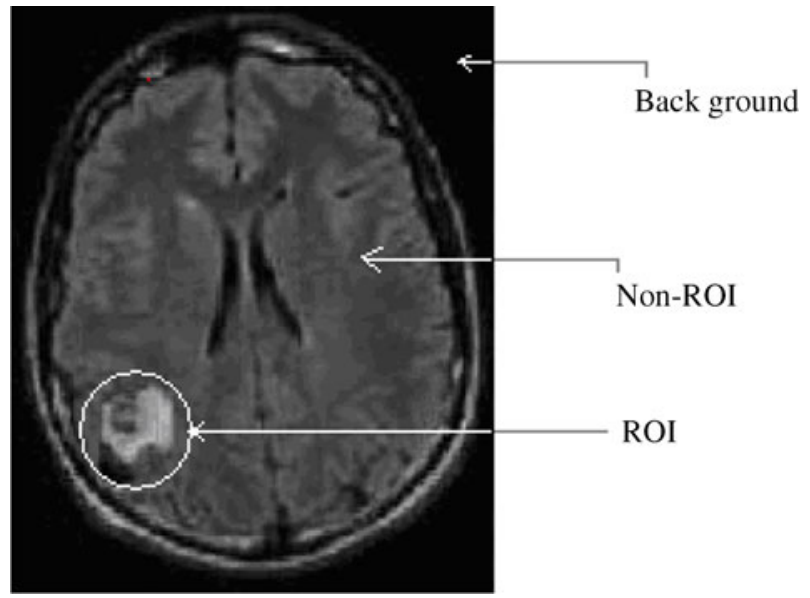

Figure 1. Different parts of the medical image.

The background is made zero using:

$$
\begin{aligned}
& \operatorname{img} g[i, j] \leq x \_ \text {th, then } \\
& \operatorname{img} g[i, j]=0 .
\end{aligned}
$$

Here, X_th is the threshold value of background of the image (img). As the background is not required reducing the background contents to zero also accounts for complete lossless compression, producing a ready to process image.

Morphological operations are effectively used, which contain a value of ' 1 ' in the foreground and a value of ' 0 ' in the background. Then the mask is logically AND-ed with the image to separate-out ROI part (IMG_ROI) and Non-ROI image part as shown in equation 2.

$$
R O I \_m a s k \& \& i m g=I M G \_R O I .
$$

The two separated parts can be processed separately as per the requirement, i.e., ROI part will be processed by lossless technique, while Non-ROI will be compressed with accepted lossy compression methods; this is shown in figure 3.

\subsection{ROI and non-ROI processing}

Lossless compression, Progressive transmission and RBC are important functionalities for a compression scheme helpful in telemedicine application (Baeza \& Verdoy 2009). User can select ROI of any arbitrary shape. ROI is compressed with lossless version of compression technique such as Huffman, Arithmetic, RLE, LZW, ZIP, etc., while Non-ROI is compressed by SPIHT

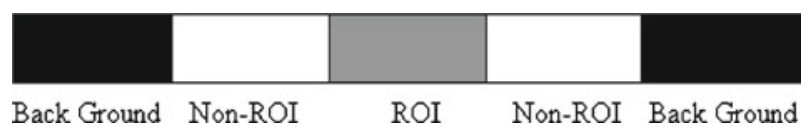

Figure 2. Cross-sectional view of medical image (statistical representation). 


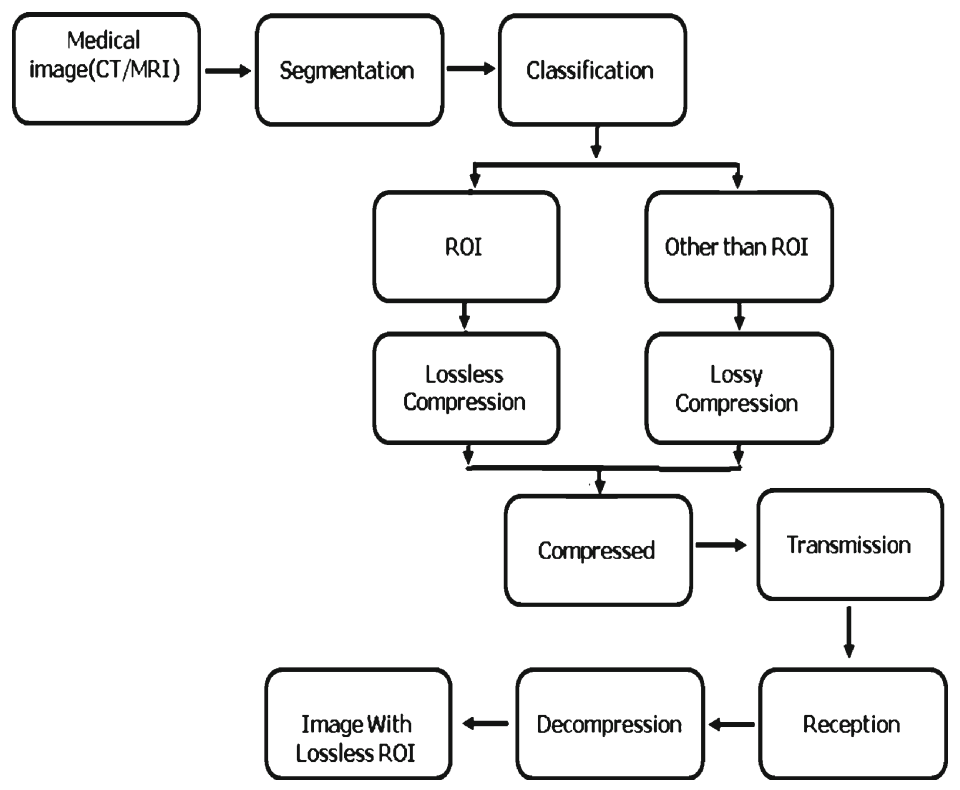

Figure 3. Block diagram of the proposed method.

algorithm used after wavelet transform. Wavelet-based techniques are the latest development in the field of image compression. It offers multi-resolution capability that is not available in any of the other methods.

\subsection{Integer wavelet transform}

The Integer Wavelet Transform (IWT) is used to have lossless processing. The wavelet transform (WT), in general, produces floating point coefficients. Although these coefficients can be used to reconstruct an original image perfectly in theory, the use of finite precision arithmetic and quantization results in a lossy scheme. Recently reversible integer wavelet transform has been introduced. Lifting provides an efficient way to implement the Discrete Wavelet Transform (DWT) and the computational efficiency of the lifting implementation can be up to $100 \%$ higher than the traditional direct convolution based implementation (Calderbank et al 1998). Lifting allows simple inverse transform of the same complexity as the forward one. Reversible IWT is composed of the elementary operations of the forward one, taken in reverse order (Reichel et al 2001). The advantages of IWT are

(i) Faster calculation with respect to traditional DWT.

(ii) Allows a fully in-place calculation of the wavelet transform, no need of temporary memory.

(iii) Generates only integer number, low computational complexity as compared to DWT which generates floating point numbers.

(iv) Completely reversible, with zero practical loss.

\subsection{Coding algorithm}

The set partition in hierarchical trees (SPIHT) coding algorithm is best in terms of compression performance (Said \& Pearlman 1996). Previously, the SPIHT was designed for lossy data 
compression. By combining the IWT with the SPIHT, both the lossy and lossless compression modes are now supported. The major advantage of using SPIHT coding technique is that, it supports embedded coding along with progressive transmission, which is suitable for telemedicine.

A new coding algorithm is presented here.

(i) Read image from database and get dimensions.

(ii) Apply threshold to remove background.

(iii) Select ROI, and separate out ROI and Non-ROI.

(iv) Accept compression levels from user.

(v) Apply wavelet for Non- ROI to execute 2D heap.

(vi) Do operation of distortion as per level selected by user for Non-ROI, and Lossless zip technique for ROI (Lehtinen 1999).

(vii) Perform inverse- wavelet recursively combine ROI.

(viii) Compare the quality of original image with newly reconstructed image.

\section{Experimental result}

Algorithm is implemented on a group of MR DICOM images. SPIHT is proved to be the best. But for ROI-based compression computational complexity is also one of the important issues to be considered, while addressing real time applications (Bhaskaranarayana et al 2009); (Park $\&$ Park 2002). A new and simple algorithm as explained above is used to encode the image. Figure 4 shows the different stages of development. Original image formatted in DICOM format of size 256 X 256 with 8 bit resolution is input to software. The 'compressed image' is the image which is generated at the decoder side after reconstruction process. The output of encoder is a bit stream of numbers arranged in a manner so as to support the progressive transmission, with initial part as a ROI compressed with run length encoding. This bit stream is transmitted over the telemedicine network using GSM mobile device.

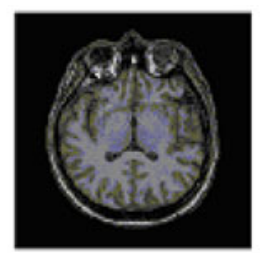

Original Image

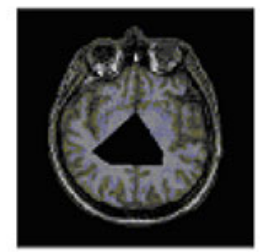

Region Other than ROI Compressed Image Image 1

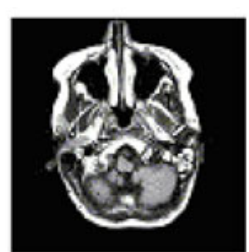

Region of Interest

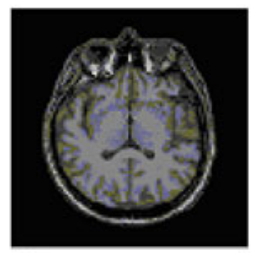

e
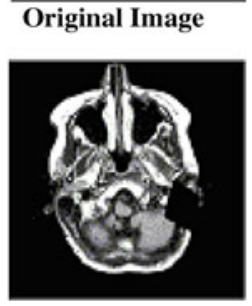

Region Other than ROI Compressed Image

Region of Interest

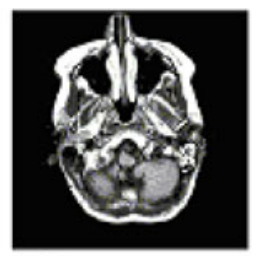

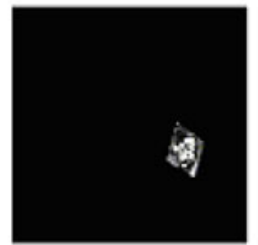

Image 2

Figure 4. Different stages of developed software for images. 
Table 1. Performance of proposed method.

\begin{tabular}{|c|c|c|c|c|c|c|c|}
\hline \multirow{2}{*}{$\begin{array}{l}\text { Sr. } \\
\text { No. }\end{array}$} & \multirow{2}{*}{$\begin{array}{l}\text { Level } \\
\text { nmax }\end{array}$} & \multicolumn{3}{|c|}{ Image 1} & \multicolumn{3}{|c|}{ Image 2} \\
\hline & & MSE & PSNR & $\mathrm{CR}$ & MSE & PSNR & CR \\
\hline 1 & 0 & 0.0005 & 80.85 & 59.93 & 0.0003 & 83.80 & 39.69 \\
\hline 2 & 1 & 0.0031 & 73.25 & 59.78 & 0.0009 & 78.46 & 39.69 \\
\hline 3 & 2 & 0.0205 & 65.02 & 58.70 & 0.0114 & 67.55 & 39.53 \\
\hline 4 & 3 & 0.2834 & 53.60 & 53.92 & 0.1338 & 56.86 & 38.00 \\
\hline 5 & 4 & 2.3427 & 44.43 & 40.98 & 0.8590 & 48.79 & 33.84 \\
\hline 6 & 5 & 17.2934 & 35.70 & 28.96 & 3.8044 & 42.30 & 26.46 \\
\hline 7 & 6 & 48.3973 & 31.28 & 20.33 & 20.8188 & 34.94 & 18.30 \\
\hline 8 & 7 & 357.641 & 22.59 & 15.71 & 36.3838 & 32.52 & 10.67 \\
\hline 9 & 8 & 704.679 & 19.65 & 14.93 & 55.5300 & 30.68 & 6.98 \\
\hline 10 & 9 & 691.057 & 19.73 & 14.91 & 153.323 & 26.32 & 6.04 \\
\hline
\end{tabular}

Table 1 shows the comparison on Mean Square Error (MSE), Peak Signal to Noise Ratio (PSNR) (calculation shown in equation 4) with respect to number of decomposition level (ITU-T recommendation H.263, 1998). Bi-orthogonal 4.4 wavelet is used in construction of 2D heap (Bairagi \& Sapkal 2009). Original image size is $64.9 \mathrm{~Kb}$ for image 1 and $65 \mathrm{~Kb}$ for image 2. But here ROI is selected and kept constant for every compression level to get unique results.

$$
\text { Bit rate }=\text { Total encoded bits } / \text { total number of bits. }
$$

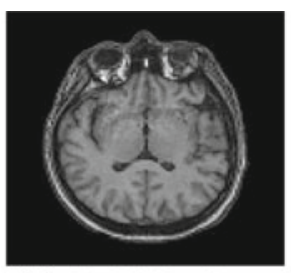

(a) LEVEL 1

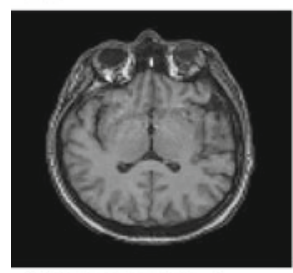

(d) LEVEL 4

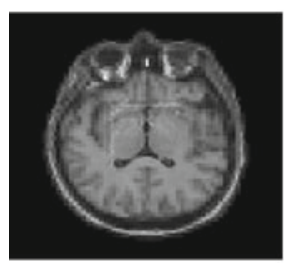

(g) LEVEL 7

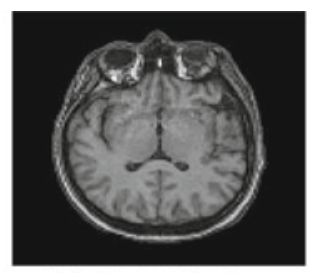

(b) LEVEL 2

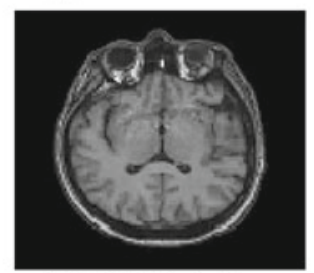

(e) LEVEL 5

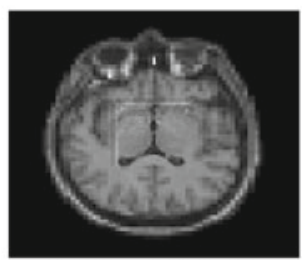

(h) LEVEL 8

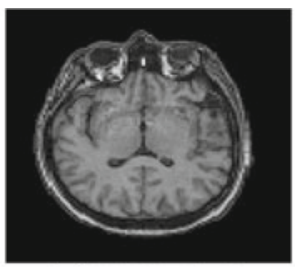

(c) LEVEL 3

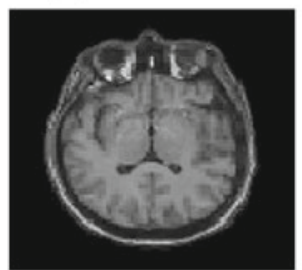

(f) LEVEL 6

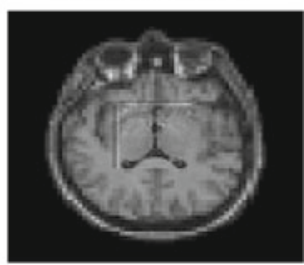

(1) LEVEL 9

Figure 5. Reconstructed images of ROI based encoding with respect to level. 


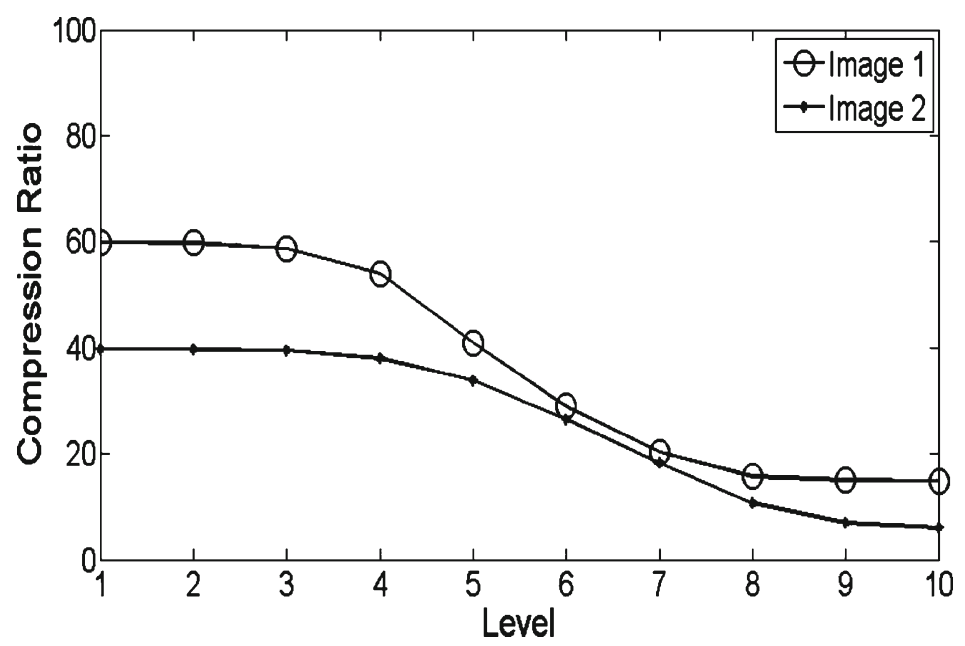

Figure 6. Analysis of compression ratio with respect to level of distortion.

$$
P S N R=10 \log \frac{M A X^{2}}{\frac{1}{w^{*} h} \sum_{i=1}^{w} \sum_{j=1}^{h}[O(i, j)-C(i, j)]^{2}} .
$$

Here, $\mathrm{O}(\mathrm{i}, \mathrm{j})$ is the original image, $\mathrm{C}(\mathrm{i}, \mathrm{j})$ is the reconstructed image, $w$ is the total number of row elements and $\mathrm{h}$ is total number of column element, MAX is 255 maximum value of Pixel for 8 bit image.

From table 1, it is clear that the horizons of the compression are now increased. Compression Ratio (CR) increases as the level increases.

Figure 5 shows the result of all nine levels. It is difficult to distinguish between ROI and Non-ROI up to level 6, but after level 7 this difference is significant.

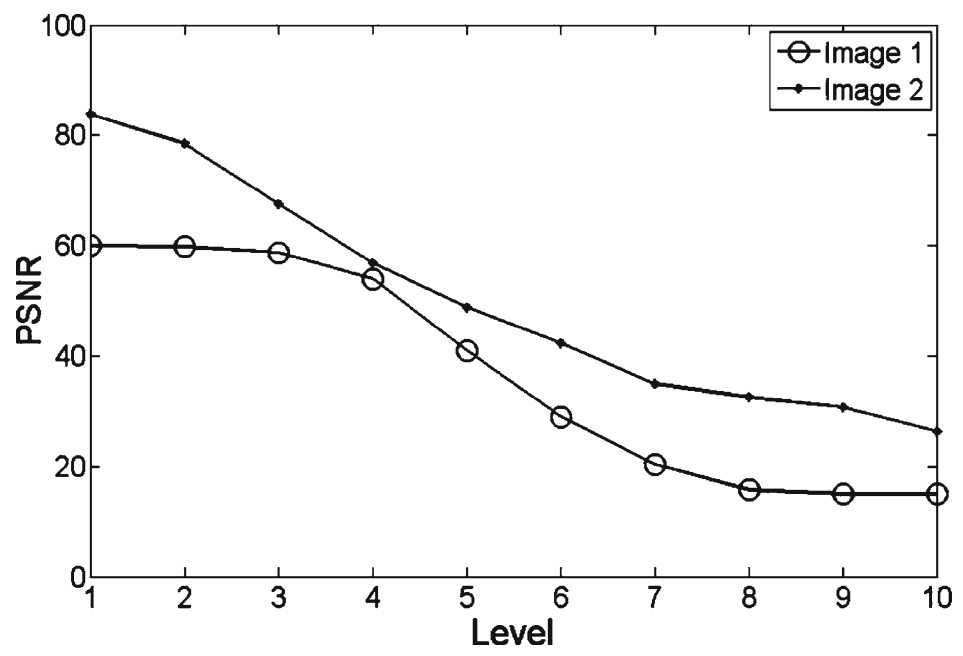

Figure 7. Analysis of PSNR with respect to level of distortion. 
There are blocking non-uniform artifacts in the image as shown in figure 5. These can be removed using smoothing filter.

From figures 6 and 7, it can be seen that the CR and PSNR values are dependent on level of distortion. As the levels increase the CR and PSNR values decrease.

\section{Comparison with other reported technique and discussion}

Several ROI-based techniques are reported and compared in (Doukas \& Maglogiannis 2007), the general scaling, the MAXSHIFT, the EZW-based, and the ROI-VQ methods require additional coefficients to decode the object. This may be the bottleneck of the system, which may increase the complexity (Maglogiannis \& Kormentzas 2009). The exact decoding of the object feasibility refers to the ability of the method to preserve the entire ROI without pixel-blending artifacts. One of the features of ROI-based coding technique is to support any arbitrary shape of ROI (Doukas $\&$ Maglogiannis 2007). The proposed technique does not require any additional bits for encoding as in MAXSHIFT. Hence, the algorithmic complexity is less, and such less complex systems are recommended for Telemedicine. Use of IWT in coding itself has reduced the complexity as compared with method in Maglogiannis \& Kormentzas (2009). Also the proposed technique supports arbitrary coding of ROI as well as progressive transmission of data with priority to ROI. Further, in the proposed technique the ROI is also compressed by lossless compression method allowing more compression. For the proposed method the computational execution time is calculated using Big-oh notation which is equals to $26 * 1+32 * \mathrm{~N}+20 * \mathrm{~N}^{2}+12 \mathrm{~N}^{3}$, therefore the computational complexity is $\mathrm{O}\left(\mathrm{N}^{3}\right)$ (Black 2008).

Different techniques can be used for Non-ROI part to compress it. There might be question that if the ROI is the only important part, then why to encode and transmit Non-ROI part? NonROI part should be encoded, because Non-ROI part gives the position of ROI in the image. Also this image is carrying the patient's other information in the text format, which is needed exclusively at the time of decoding the image. Also when ROI based coding is used, it is not fruitful to use conventional objective quality analysis measures. Because the ROI part is encoded by lossless compression technique and only Non-ROI part is to be examined. Hence, there is a need for new objective measures to address this issue.

\section{Conclusion}

Every image contains some redundant information, which needs to be identified by the user to obtain compression. The floating point representation of the DWT gives small error in the system. The IWT is recommended for critical medical application because of its perfect reconstruction property. ROI-based compression is providing better results as compared with lossless methods, along with preservation of diagnostically important information. Such method is recommended for telemedicine system especially rural area, where network resources have limitations. Advanced version of the proposed method may include the compression based on the information contents as well as compression based on ROI to be selected automatically.

\section{Acknowledgements}

The authors would like to thank the Pune University, Pune, India for financially supporting this work under research grant and also thank Sinhgad General Hospital, Pune for valuable help and 
support. The authors would also like to thank reviewers of this paper for useful comments which helped to improve the paper.

\section{References}

Ali T J and Akhtar P 2008 Significance of region of interest applied on MRI and CT images in teleradiologytelemedicine. Heidelberg: Springer-Verlag Berlin, 151-159

Baeza I and Verdoy A 2009 ROI-based Procedures for progressive transmission of digital images: A comparison. Elsevier J. Math. Comput. Model. 50: 849-859

Bairagi V K and Sapkal A M 2009 Selection of Wavelets for Medical Image Compression. IEEE Int Conf ACT 2009, Trivendrum, India, 678-680

Bhaskaranarayana A, Satyamurthy L S, Murthy L N R, Sethuraman K and Rayappa H 2009 Bridging health divide between rural and urban areas - Satellite based telemedicine networks in India. Space Technologies for the Benefit of Human Society and Earth Part II, P. Olla (ed), Netherlands: Springer Book Series, $160-178$

Black P E 2008 big-O notation, Dictionary of algorithms and data structures, P E Black (ed), U.S. National Institute of Standards and Technology. http://www.itl.nist.gov/div897/sqg/dads/HTML/ bigOnotation.html

Calderbank A R, Daubechies I, Sweldens W and Yeo B L (1998) Wavelet transforms that map integers to integers. Appl. Comput. Harmon. Anal. 5: 332-369

Doukas C and Maglogiannis I 2007 Region of Interest Coding Techniques for Medical Image Compression. IEEE Eng. Med. Biol. Mag. 26(5): 29-35

ITU Telecom, Standardization Sector of ITU 1998 Video Coding for Low Bitrate Communication. Draft ITU-T Recommendation H.263 Ver 2

Lehtinen J 1999 Limiting distortion of a wavelet image codec. J. Acta Cyberntica. 14: 341-356

Maglogiannis I and Kormentzas G 2009 Wavelet-based compression with ROI coding support for mobile access to DICOM images over heterogeneous radio networks. Trans. Inform. Technol. Biomed. 13(4): 458-466

Miaou S G, Ke F S and Chen S C 2009 A lossless compression method for medical image sequences using JPEG-LS and interframe coding. IEEE Trans. Inform. Technol. Biomed. 13(5): 818-821

Park K H and Park H W 2002 Region-of-interest coding based on set partitioning in hierarchical trees. IEEE Trans. Circuits Syst. Video Technol. 12(2): 106-113

Placidi G 2009 Adaptive compression algorithm from projections: Application on medical greyscale images. J. Comput. Biol. Med. 39: 993-999

Reichel J, Menegaz G, Nadenau M J and Kunt M 2001 Integer Wavelet Transform for Embedded Lossy to Lossless Image Compression. IEEE Trans. Image Process 10930: 383-392

Said A and Pearlman W A 1996 A new, fast and efficient image codec based on set partitioning in hierarchical trees. IEEE Trans. Circuits Syst. Video Technol. 6: 243-250

Sanchez V, Abugharbieh R and Nasiopoulos P 2009 Symmetry-Based Scalable Lossless Compression of 3D Medical Image Data. IEEE Trans. Med. Imaging 28(7): 1062-1071 